\title{
Lane Keeping Control Based on Double Integration and Bipolar Function
}

\author{
Junbo Zhao ${ }^{1}$, Jie Yang ${ }^{1}$ and Huali $\mathrm{Wu}^{2}$ \\ ${ }^{1}$ Zhejiang Institute of Communications, Hang Zhou, 311112, China \\ ${ }^{2}$ Department of Control Engineering, Naval Aeronautical and Astronautical \\ University, Yantai, 264001 \\ 1yangjie@zjvtit.edu.com, ${ }^{2}$ tom_linhuali@126.com
}

\begin{abstract}
The lane keeping problem of automatic driving for vehicles was studied based on the position movement equation of simplified linear lateral dynamic model. Two kinds of novel sliding mode surface were constructed by introducing double integration and bipolar function. Also adaptive laws were designed to solve the uncertain problem of system model and Lyapunov function was constructed to prove the stability of the above two sliding mode lane keeping methods. At last, detailed random numerical simulations were done and simulation results were compared with other three methods to testify the effectiveness of the proposed method.
\end{abstract}

Keywords: Lane keeping; traffic engineering; sliding mode control; adaptive; double integration; bipolar function

\section{Introduction}

In recent years, the scholars at home and abroad have been attracted by the related technology of automatic driving for vehicles, whose control including lateral control and longitudinal control. By the offset between the actual direction of vehicle moving and the central direction of the lane, this technology calculates the corner of the steering wheel with corresponding control algorithm, then based on which the vehicle is controlled to drive safely following the lane. The difficulty for control algorithm of lane keeping lied in the adaptability for uncertain cases in vehicle driving, such as unknown road curvature, wind disturbance, inaccurate modeling of pavement and tire friction. For the above uncertain problems, many control methods, such as PID control, adaptive control, variable structure control, neural network control and so on, had been widely used in land keeping of vehicle driving, and achieved good results. Among these methods, sliding mode control had attracted more scholars because of its good robustness and simplicity during design produced by order-reduce in system.

In reference [6] etc., based on traditional sliding mode control, a kind of self tuning sliding mode method on the basis of Sigmoid function was proposed to eliminate the flutter phenomenon in the control of lateral position in vehicles. In reference [7] etc., considering uncertain cases such as the mass of the vehicle, the moment of inertia and so on, a kind of quasi-sliding mode control was designed to solve two situations, one was the parameters of model varying with time bounded, the other was the parameters slowly varying unknown. In reference [8] etc., for non-matching uncertainty and time-delay in state when keeping the lane, a kind of self tuning sliding mode keeping method was designed by adopting LMI theory to calculate the sufficient condition for existence of the sliding mode. In reference [9] etc., when keeping the lane in uncertain automated highway system, a kind of sliding mode surface with Terminal type was introduced to design the terminal sliding mode controller to solve the problem of uncertain model parameters. In reference [10] etc., because the control algorithm of lane keeping possessed inadequate 
adaptability on the perturbation of parameters, thus a kind of sliding mode control method based on fuzzy adaptive adjustment was introduced to improve its robustness for parameter perturbation and un-modeled dynamics. In reference [11] etc., a kind of adaptive sliding mode control method was put forward. It was applied to the control of lane keeping in intelligent vehicle to solve the problems of the known external disturbance, matching uncertainty and time-delayed state. In reference [12] etc., based on the Terminal sliding mode surface, a kind of direct adaptive method was proposed to solve the problem of parameters uncertainty. This paper based on the above literatures, raised two new sliding mode surface, in which the new double integral sliding mode surface could improve the control precision by using the integrator, the other bio-polar sliding mode could lead Sigmoid function into both sliding mode surface and control variable to effectively reduce the flutter problem in traditional sliding mode.

\section{Model Description}

On the premise of assuming the rotation angle of wheel as a small angle, the lateral position error and the lateral yaw angle error were chosen as the basic state variables to describe the model of lateral motion for vehicles. The mode was written as the following [13-14] formula:

$$
\begin{gathered}
\ddot{y}=-\frac{2\left(C_{f}+C_{r}\right)}{m v_{x}} \dot{y}-\left[v_{x}+\frac{2\left(C_{f} l_{f}-C_{r} l_{f}\right)}{m v_{x}}\right] \dot{\psi}+\frac{2 C_{f}}{m} \delta_{z} \\
\ddot{\psi}=-\frac{2\left(C_{f} l_{f}^{2}+C_{r} l_{r}^{2}\right)}{I_{z} v_{x}} \dot{\psi}-\frac{2\left(C_{f} l_{f}-C_{r} l_{r}\right)}{I_{z} v_{x}} \dot{y}+\frac{2 C_{f} l_{f}}{I_{z}} \delta_{z}
\end{gathered}
$$

In which, $\psi$ was shown as the lateral yaw angle; $y$ was shown as the lateral position; $I_{z}$ was described as the moment of inertia for vehicle; $v_{x}$ was indicated as the longitudinal velocity for vehicle; $m$ was meant for the mass of the vehicle; $l_{f}$ and $l_{r}$ were shown as the distance from the centre of mass to the front axle and the back axle respectively in vehicle; $C_{f}$ and $C_{r}$ were written as the stiffness of the front wheels and the back wheels; $\delta$ was shown as the rotating angle of the front wheels.

Referenced as literature [12], if exploration strategy was assumed to be used, then two lateral sensors were installed at the front and rear bumpers. In this way, the lateral offset of the wheel from the central line and the angle between the moving direction of vehicle and the tangent of the path could be both measured. Let the explored distance be taken as $d$, the lateral moving model in geometry of vehicles could be shown as follows:

$$
\begin{aligned}
& \dot{y}_{s}=\dot{y}+v_{x} \psi_{r}+d \dot{\psi}_{r} \\
& \ddot{\psi}_{r}=\ddot{\psi}-\ddot{\psi}_{d}
\end{aligned}
$$

In which, $\psi_{r}$ was shown as the lateral yaw angle error, that was the angle between the moving direction of vehicles and the tangent of the path; $y_{s}$ was shown as the lateral position error off the central line; $\psi_{d}$ was the desired yaw angle, $\chi$ was the curvature of the center line in the lane, that the equation $\mathrm{t} \dot{\psi}_{d}=v_{x} \chi$ could be obtained.

\section{Transformation and Hypothesis of the Model}

According to equations (1) to (4), the related variables were defined as follows:

$$
a_{1}=C_{f}+C_{r}, \quad a_{2}=C_{f} l_{f}-C_{r} l_{r}, a_{3}=C_{f} l_{f}^{2}+C_{r} l_{r}^{2}
$$




$$
\begin{gathered}
a_{11}=\frac{2 a_{2}}{I_{z}}, \quad a_{12}=2\left[\frac{d a_{2}}{I_{z} v_{x}}-\frac{a_{3}}{I_{z} v_{x}}\right] \\
a_{13}=-\frac{2 a_{2}}{I_{z} v_{x}}, \quad b_{1}=-\frac{2 C_{f} l_{f}}{I_{z}}, \quad d_{1}=-\frac{2 a_{3}}{I_{z} v_{x}} \dot{\psi}_{d}-\ddot{\psi}_{d} \\
a_{21}=2\left[\frac{a_{1}}{m}+\frac{d a_{2}}{I_{z}}\right], \quad a_{22}=2\left[\frac{d a_{1}}{m v_{x}}-\frac{a_{2}}{m v_{x}}+\frac{d^{2} a_{2}}{I_{z} v_{x}}-\frac{d a_{3}}{I_{z} v_{x}}\right] \\
a_{23}=-2\left[\frac{a_{1}}{m v_{x}}+\frac{d a_{2}}{I_{z} v_{x}}\right], \quad b_{2}=2\left[\frac{C_{f}}{m}+\frac{d C_{f} l_{f}}{I_{z}}\right] \\
d_{2}=-\left[v_{x}+\frac{2 a_{2}}{m v_{x}}+\frac{2 d a_{3}}{I_{z} v_{x}}\right] \dot{\psi}_{d}-d \ddot{\psi}_{d}
\end{gathered}
$$

Then the lateral moving model of vehicles could be described as the following form:

$$
\left[\begin{array}{c}
\ddot{\psi}_{r} \\
\ddot{y}_{s}
\end{array}\right]=\left[\begin{array}{llll}
a_{11} & a_{12} & 0 & a_{13} \\
a_{21} & a_{22} & 0 & a_{23}
\end{array}\right]\left[\begin{array}{c}
\psi_{r} \\
\dot{\psi}_{r} \\
y_{s} \\
\dot{y}_{s}
\end{array}\right]+\left[\begin{array}{l}
b_{1} \\
b_{2}
\end{array}\right] \delta_{z}+\left[\begin{array}{l}
d_{1} \\
d_{2}
\end{array}\right]
$$

With $\dot{\psi}_{r}=\omega$ and $\dot{y}_{s}=v_{y}$ defined, the above model could be written as standard state-variable mode.

$$
\left[\begin{array}{c}
\dot{\psi}_{r} \\
\dot{\omega} \\
\dot{y}_{s} \\
\dot{v}_{y}
\end{array}\right]=\left[\begin{array}{cccc}
0 & 1 & 0 & 0 \\
a_{11} & a_{12} & 0 & a_{13} \\
0 & 0 & 0 & 1 \\
a_{21} & a_{22} & 0 & a_{23}
\end{array}\right]\left[\begin{array}{c}
\psi_{r} \\
\omega \\
y_{s} \\
v_{y}
\end{array}\right]+\left[\begin{array}{c}
0 \\
b_{1} \\
0 \\
b_{2}
\end{array}\right] \delta_{z}+\left[\begin{array}{c}
0 \\
d_{1} \\
0 \\
d_{2}
\end{array}\right]
$$

Referenced by the literature [10], it was considerated further the characteristic of inertial lag from the steering to the front wheels angle $\delta_{z}$. The first-order inertial component was introduced to describe its dynamic characteristic shown as below:

$$
\dot{\delta}=\frac{1}{T_{e}} u-\frac{1}{T} \delta
$$

Considering the practice and complexity in the problem of lane keeping, the hypothesis was made for model with reference to literature [11].

HYPOTHESIS 1: The variables $m, I_{z}, l_{f}, l_{r}, C_{f}$ and $C_{r}$ of vehicles are all unknown.

HYPOTHESIS 1: The curvature $\chi$ of the pavement was also the unknown parameter, but its value was bounded.

\section{Design of Double Integral Sliding Mode Controller}

Without loss of generality, the expected value of the lateral position error $y_{s}$ was defined as $y_{r \text { in }}$. And without special introductions, $y_{r \text { in }}=0$. The error variable was defined as $e_{y}=y_{s}-y_{\text {rin }}$, then the following equation could be obtained.

$$
\dot{e}_{y}=\dot{y}_{s}-\dot{y}_{\text {rin }}
$$


The sliding surface was chosen as below

$$
s_{y}=\dot{e}_{y}+c_{1} e_{y}+c_{2} \int e_{y} d t+c_{3} \iint e_{y} d t d t
$$

The derivation of the equation (15) was written as

$$
\dot{s}_{y}=\ddot{e}_{y}+c_{1} \dot{e}_{y}+c_{2} e_{y}+c_{3} \int e_{y} d t
$$

The above equations could be deduced to the following equation.

$$
\begin{aligned}
\dot{s}_{y} & =\ddot{y}_{s}+c_{1} \dot{y}_{s}+c_{2} e_{y}+c_{3} \int e_{y} d t \\
& =\dot{v}_{y}+c_{1} v_{y}+c_{2} e_{y}+c_{3} \int e_{y} d t \\
& =a_{21} \psi_{r}+a_{22} \omega+\left(a_{23}+c_{1}\right) v_{y}+c_{2} e_{y}+b_{2} \delta+d_{2}+c_{3} \int e_{y} d t
\end{aligned}
$$

Corresponding to the calculation of practical parameters, $b_{2}>0$, so equation (17) could be written as follows further.

$$
\frac{1}{b_{2}} \dot{s}_{y}=\frac{a_{21}}{b_{2}} \psi_{r}+\frac{a_{22}}{b_{2}} \omega+\frac{\left(a_{23}+c_{1}\right)}{b_{2}} v_{y}+\frac{c_{2}}{b_{2}} e_{y}+\frac{d_{2}}{b_{2}}+\frac{c_{3}}{b_{2}} \int e_{y} d t+\delta
$$

First considering the certain case, that was parameters of the vehicle model and the curvature of the path were known completely, the double integral sliding mode control law could be designed as below:

$$
\delta_{1}=-\frac{a_{21}}{b_{2}} \psi_{r}-\frac{a_{22}}{b_{2}} \omega-\frac{\left(a_{23}+c_{1}\right)}{b_{2}} v_{y}-\frac{c_{2}}{b_{2}} e_{y}-\frac{d_{2}}{b_{2}}-\frac{c_{3}}{b_{2}} \int e_{y} d t+\Omega_{1}
$$

Among which:

$$
\Omega_{1}=-k_{1} s-\frac{k_{2} s}{|s|+\varepsilon}-k_{3} \frac{1-e^{-\tau s}}{1+e^{-\tau s}}
$$

Among which, $k_{2}+k_{3}$ was the physical limitation of maximum steering angle for front wheels. For example, if the maximum value of steering angle was limited as $15^{\circ}$, $k_{2}$ and $k_{3}$ could be set as $k_{2}=7 / 57.3, k_{3}=8 / 57.3$.

When the parameters of model for vehicle were unknown completely, adaptive double integral sliding mode control law could be designed as follows.

$$
g_{1}=\frac{a_{21}}{b_{2}}, g_{2}=\frac{a_{22}}{b_{2}}, g_{3}=\frac{\left(a_{23}+c_{1}\right)}{b_{2}}, g_{4}=\frac{c_{2}}{b_{2}}, g_{5}=\frac{d_{2}}{b_{2}}, g_{6}=\frac{1}{b_{2}}
$$

Design $\delta_{2}$ as below:

$$
\delta_{2}=-\hat{g}_{1} \psi_{r}-\hat{g}_{2} \omega-\hat{g}_{3} v_{y}-\hat{g}_{4} e_{y}-\hat{g}_{5}-\hat{g}_{6} \int e_{y} d t+\Omega_{1}
$$

Error variables were defined as the following:

$$
\begin{aligned}
& \tilde{g}_{1}=g_{1}-\hat{g}_{1}, \quad \tilde{g}_{2}=g_{2}-\hat{g}_{2}, \quad \tilde{g}_{3}=g_{3}-\hat{g}_{3} \\
& \tilde{g}_{4}=g_{4}-\hat{g}_{4}, \quad \tilde{g}_{5}=g_{5}-\hat{g}_{5}, \quad \tilde{g}_{6}=g_{6}-\hat{g}_{6}
\end{aligned}
$$

Then we could get the equation as: 


$$
\frac{1}{b_{2}} \dot{s}_{y}=\tilde{g}_{1} \psi_{r}+\tilde{g}_{2} \omega+\tilde{g}_{3} v_{y}+\tilde{g}_{4} e_{y}+\tilde{g}_{5}+\tilde{g}_{6} \int e_{y} d t+\Omega_{1}
$$

The regulation rules of adaptive weights for unknown parameters were designed as below equations.

$$
\begin{gathered}
\dot{\hat{g}}_{1}=-\Gamma_{1} s_{y} \psi_{r}, \quad \dot{\hat{g}}_{2}=-\Gamma_{2} s_{y} \omega, \quad \dot{\hat{g}}_{3}=-\Gamma_{3} s_{y} v_{y} \\
\dot{\hat{g}}_{4}=-\Gamma_{4} s_{y} e_{y}, \quad \dot{\hat{g}}_{5}=-\Gamma_{5} s_{y}, \quad \dot{\hat{g}}_{6}=-\Gamma_{6} s_{y} \int e_{y} d t
\end{gathered}
$$

Chosen Lyapunov function as below:

$$
V=\frac{b_{2}}{2} s_{y}^{2}+\sum_{i=1}^{6} \frac{1}{\Gamma_{i}} \tilde{g}_{i}^{2}
$$

Find the derivation of this function, we got:

$$
\dot{V}=-s_{y} \Omega_{1} \leq 0
$$

It was visible that system was stable according to the theory of Lyapunov stability, thus $e_{y} \rightarrow 0$, the control of lane keeping was realized.

\section{Design of Bipolar Sliding Mode Controller}

The bipolar sliding mode surface could be also defined as follows:

$$
S_{y}=\dot{e}_{y}+c_{1} \frac{1-e^{-\tau e_{y}}}{1+e^{-\tau e_{y}}}
$$

The derivation of the equation (30) was written as

$$
\dot{s}_{y}=\ddot{e}_{y}+c_{1}\left(\frac{1-e^{-\tau e_{y}}}{1+e^{-\tau e_{y}}}\right)^{\prime}
$$

Simplified equation (31) further, the following equation was obtained.

$$
\dot{s}_{y}=a_{21} \psi_{r}+a_{22} \omega+a_{23} v_{y}+c_{2} e_{y}+b_{2} \delta+d_{2}+c_{1} \Lambda
$$

Among which,

$$
\Lambda=\left(\frac{1-e^{-\tau e_{y}}}{1+e^{-\tau e_{y}}}\right)^{\prime}=\frac{2 e^{-\tau e_{y}}}{\left(1+e^{-\tau e_{y}}\right)^{2}} \tau \dot{e}_{y}=\frac{2 e^{-\tau e_{y}}}{\left(1+e^{-\tau e_{y}}\right)^{2}} \tau v_{y}
$$

Corresponding to the calculation of practical parameters, $b_{2}>0$, so equation (32) could be written as follows further.

$$
\frac{1}{b_{2}} \dot{s}_{y}=\frac{a_{21}}{b_{2}} \psi_{r}+\frac{a_{22}}{b_{2}} \omega+\frac{a_{23}}{b_{2}} v_{y}+\frac{c_{2}}{b_{2}} e_{y}+\frac{d_{2}}{b_{2}}+\frac{2 e^{-\tau e_{y}}}{b_{2}\left(1+e^{-\tau e_{y}}\right)^{2}} \tau v_{y}+\delta
$$

Considering the vehicle model was accurate completely, the following control law could be designed.

$$
\delta_{1}=-\frac{a_{21}}{b_{2}} \psi_{r}-\frac{a_{22}}{b_{2}} \omega-\frac{a_{23}}{b_{2}} v_{y}-\frac{c_{2}}{b_{2}} e_{y}-\frac{d_{2}}{b_{2}}-\frac{2 e^{-\tau e_{y}}}{b_{2}\left(1+e^{-\tau e_{y}}\right)^{2}} \tau v_{y}+\Omega_{1}
$$


Among which, the definition of $\Omega_{1}$ was the same as above. When parameters of vehicle were unknown, we had these definition as below:

$$
g_{a 1}=\frac{a_{21}}{b_{2}}, g_{a 2}=\frac{a_{22}}{b_{2}}, g_{a 3}=\frac{a_{23}}{b_{2}}, g_{a 4}=\frac{c_{2}}{b_{2}}, g_{a 5}=\frac{d_{2}}{b_{2}}, g_{a 6}=\frac{1}{b_{2}}
$$

Adaptive bipolar sliding mode control law was designed as :

$$
\delta_{2}=-\hat{g}_{a 1} \psi_{r}-\hat{g}_{a 2} \omega-\hat{g}_{a 3} v_{y}-\hat{g}_{a 4} e_{y}-\hat{g}_{a 5}-\hat{g}_{a 6} \frac{2 e^{-\tau e_{y}}}{\left(1+e^{-\tau e_{y}}\right)^{2}} \tau v_{y}+\Omega_{1}
$$

The error variables were defined as following:

$$
\begin{array}{ccc}
\tilde{g}_{a 1}=g_{a 1}-\hat{g}_{a 1}, & \tilde{g}_{a 2}=g_{a 2}-\hat{g}_{a 2}, & \tilde{g}_{a 3}=g_{a 3}-\hat{g}_{a 3} \\
\tilde{g}_{a 4}=g_{a 4}-\hat{g}_{a 4}, & \tilde{g}_{a 5}=g_{a 5}-\hat{g}_{a 5}, & \tilde{g}_{a 6}=g_{a 6}-\hat{g}_{a 6}
\end{array}
$$

Then we would get the equation:

$$
\frac{1}{b_{2}} \dot{s}_{y}=\tilde{g}_{a 1} \psi_{r}+\tilde{g}_{a 2} \omega+\tilde{g}_{a 3} v_{y}+\tilde{g}_{a 4} e_{y}+\tilde{g}_{a 5}+\tilde{g}_{a 6} \frac{2 e^{-\tau e_{y}}}{\left(1+e^{-\tau e_{y}}\right)^{2}} \tau v_{y}+\Omega_{1}
$$

The regulation rules of adaptive weights for unknown parameters were designed as below equations.

$$
\begin{gathered}
\dot{\hat{g}}_{a 1}=-\Gamma_{1} s_{y} \psi_{r}, \quad \dot{\hat{g}}_{a 2}=-\Gamma_{2} s_{y} \omega, \quad \dot{\hat{g}}_{a 3}=-\Gamma_{3} s_{y} v_{y} \\
\dot{\hat{g}}_{a 4}=-\Gamma_{4} s_{y} e_{y}, \quad \dot{\hat{g}}_{a 5}=-\Gamma_{5} s_{y}, \quad \dot{\hat{g}}_{a 6}=-\Gamma_{6} s_{y} \frac{2 e^{-\tau e_{y}}}{\left(1+e^{-\tau e_{y}}\right)^{2}} \tau v_{y}
\end{gathered}
$$

Chosen Lyapunov function as below:

$$
V=\frac{b_{2}}{2} s_{y}^{2}+\sum_{i=1}^{6} \frac{1}{\Gamma_{i}} \tilde{g}_{a i}^{2}
$$

Find the derivation of this function, we got:

$$
\dot{V}=-s_{y} \Omega_{1} \leq 0
$$

It was visible that system was stable according to the theory of Lyapunov stability, thus $e_{y} \rightarrow 0$, the control of lane keeping was realized.

\section{Simulation and Analysis}

The parameters were assigned as the following value: the mass of the vehicle $m=1573 \mathrm{~kg}$; the moment of inertia $I_{z}=2873 \mathrm{~kg} \cdot \mathrm{m}^{2}$; the front wheels base $l_{f}=1.1 \mathrm{~m}$; the rear wheelbase $l_{r}=1.58 \mathrm{~m}$; the stiffness of the front wheels $C_{f}=80 \mathrm{kN} / \mathrm{rad}$ and the stiffness of the rear wheel $C_{r}=80 \mathrm{kN} / \mathrm{rad}$; the longitudinal velocity of vehicle was $20 \mathrm{~m} / \mathrm{s}$; the delayed time constant for the steering $T_{e}=0.05 \mathrm{~s}$; the initial error of the yaw angle was 0 degree; the initial error of the lateral position was $1 \mathrm{~m}$; other initial states were considered as 0 . 
The following Figure 1 and Figure 2 showed the control effect of traditional sliding mode control and self-tuning sliding mode control in paper [12]. Figure 3 and Figure 4 gave the control effect of the direct adaption elaborated in literature [11].

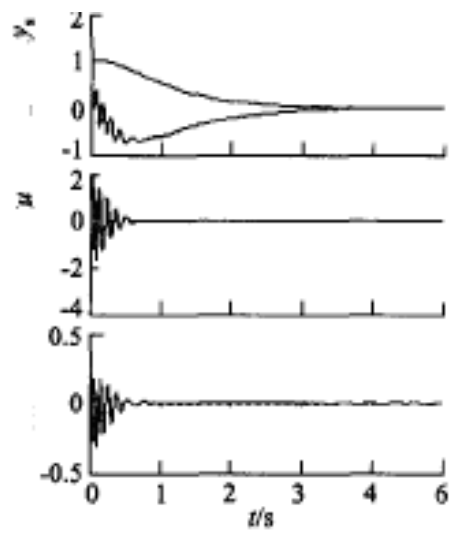

Figure 1. Curve of Traditional Sliding Mode Control

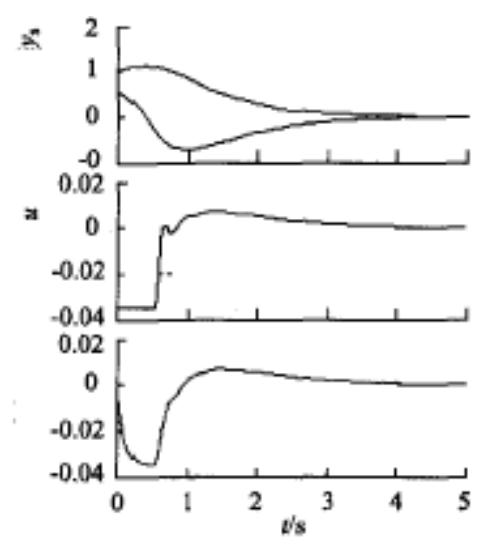

Figure 2. Curve of Adaptive Sliding Mode Control

It could be seen from Figure 1, the convergence time of traditional sliding mode control was about $3 \mathrm{~s}$, the maximum steering angle of wheel reached to more than $17^{\circ}$; while from Figure 2, the convergence time of self-turning sliding mode control is about $3 \mathrm{~s}$, but the maximum steering angle of wheel was only about $2^{\circ}$. Of course the parameters of vehicles in Figure 1 and Figure 2 are slightly different from the parameters adopted in the following Figure 3 and Figure 4.

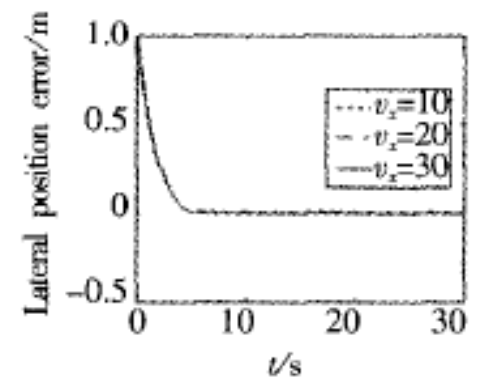

Figure 3. Curve of Direct Adaptive Control 


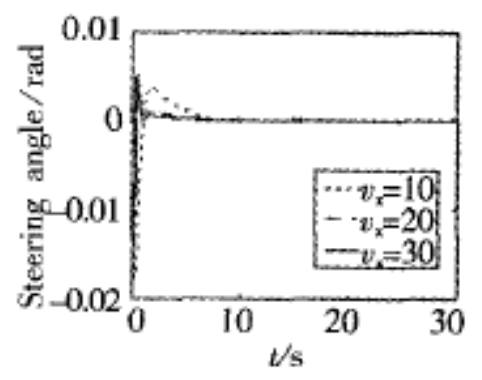

Figure 4. Turning Angle of Direct Adaptive Control

The following Figure 5 and Figure 6 were shown the control effect for the first kind of integral sliding mode control designed in this paper. In this simulation, nominal value was adopted in control law and random perturbation was executed for parameters in vehicle mode. Simulation were repeated 50 times, with the stiffness of the tires $C_{f}$ and $C_{r}$ chosen as the random values in the region $[40,80]$, the curvature radius of the path selected as the random value in the range of $[100,500]$. The results were shown in the following Figures.

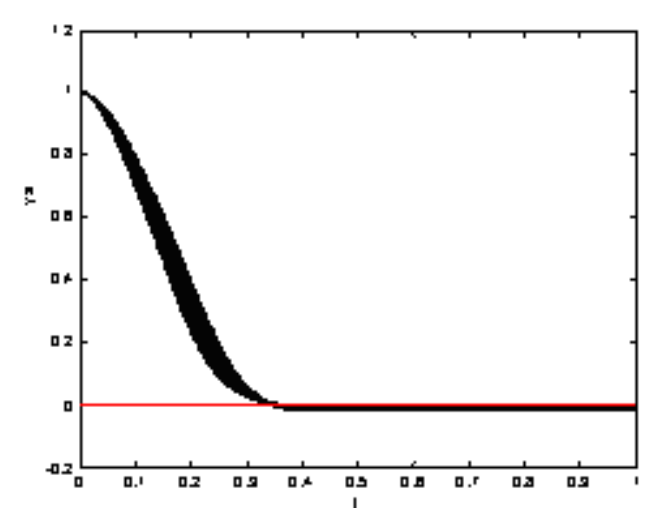

Figure 5. Curve of Lateral Position Error

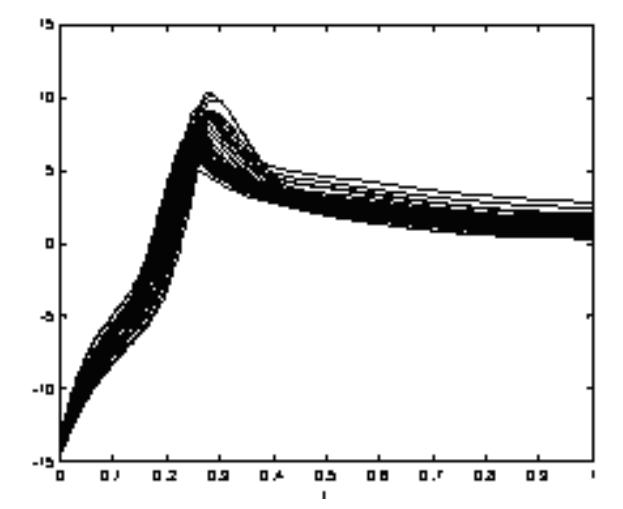

Figure 6. Curve of the Front Tyre Angle

Parameter perturbation was selected as the same above, control variable was also chosen the same as the second kind of bipolar sliding mode control method. Figure 7 and Figure 8 showed the control effect for the second kind of anti-saturation sliding mode control law. 


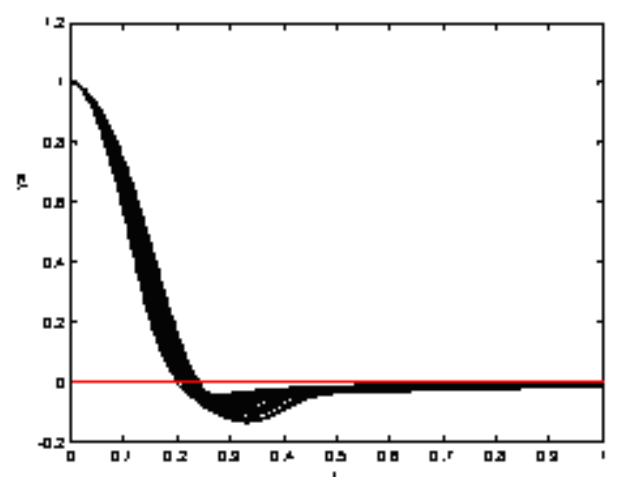

Figure 7. Curve of Lateral Position Error

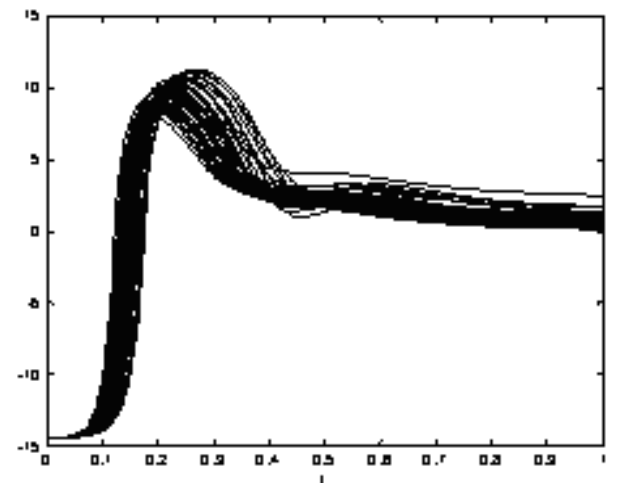

Figure 8. Curve of the Front Tyre Angle

From the above simulation and analysis, it was shown that double integral sliding mode adaptive control and bipolar adaptive sliding mode control proposed in this paper had better rapidity, their converged time was about $0.5 \mathrm{~s}$. However, methods introduced in literature [12], as traditional sliding mode control and self-tuning adaptive sliding mode control, had 3s converged time. While the direct adaptive method introduced in literature [11] had 6s converged time. At the same time, from the view of the steering angle of front wheels, the maximum angle of direct adaptive sliding mode control was $1^{\circ}$, but with slow converged velocity. According to the methods introduced in this paper, the steering angle of front wheels would be smaller than $15^{\circ}$, which satisfied the expected design. And under random perturbation, the control law could made the steering angle of front wheels satisfied the limitation strictly. In fact, reducing $k_{2}$ and $k_{3}$ could reduce the steering angle of front wheels with sacrificing the rapidity response. During practical using, values of parameters could be selected for a compromise according to requirements. It was worth mentioning that bipolar adaptive sliding mode control had better rapidity than double integral sliding mode adaptive control, but also with a little higher overshoot.

\section{Conclusion}

For the moving equation of centre of mass in simplified linear model of lateral dynamics for vehicles, double integral and bipolar adaptive sliding mode control methods were presented in this paper. In the case of unknown parameters in vehicle model, by constructing Lyapunov function and designing adaptive law of unknown parameters, both two methods could eliminate lateral position error automatically with quick speed. It was worth mentioning that, because of clever construction for double integral and bipolar sliding mode, both two methods had favorable rapidity, and the feature of steering angle of front wheels satisfying expected limitation strictly. At the end of this paper, detailed random simulation and analysis were given for multi-cases of unknown parameters in 
model. And the methods proposed in this paper was verified availability compared with other three sliding mode control methods shown in before literatures.

\section{References}

[1] W. Jingqi, C. Huiyan and Z. Pei, "Application of fuzzy adaptive PID and previewing algorithm in steering control of autonomous land vehicle", Automotive Engineering, vol. 25, no. 4, (2003), pp. 367-371.

[2] W. Rongben, L. Bing and X. Youchun, "Optimal controller design for an intelligent vehicle based on vision navigation", Automotive Engineering, vol. 23, no. 2, (2001), pp. 97-100.

[3] G. Zhenhai, "Vehicle direction preview adaptive PD control algorithm", Chinese Journal of Mechnical Engneering, vol. 40, no. 5, (2004), pp. 101-105.

[4] D. Haitao, G. Konghui and L. Fei, "Arbitrary path and speed following driver model based on vehicle acceleration feedback", Journal of Mechnical Engneering, vol. 46, no. 10, (2010), pp. 116-120.

[5] L. Cai, A. B. Rad and W. L. Chan, "Fuzzy PD controller for automatic steering control of autonomous vehicles", Proceedings of the IEEE International Conference on Fuzzy Systems, (2003), pp. 549-554.

[6] W. Li, J. Wang and J. Duan, "Self-tuning sliding mode control for vehicle's lane keeping", Journal of Highway and Transportation Research and Development, vol. 28, no. 6, (2011), pp. 136-140.

[7] R. Dianbo, C. Shengmin and Z. Guanzhe, "Quasi-sliding mode control for lane keeping with bounded and varying parameters", Transactions of Beijing institute of technology, vol. 31, no. 1, (2011), pp. 69-73.

[8] L. Wei, D. Jian-min and G. Jian-wei, "Vehicle lane-keeping control under state time-delay and mismatched uncertain perturbations", Control Theory \& Applications, vol. 29, no. 3, (2012), pp. 389-394.

[9] Z. Jiye and R. Dianbo, "Terminal Sliding Mode Control for Lane Keeping with Parametric Uncertainties", Journal of Dalian Maritime University, vol. 34, no. 3, (2008), pp. 81-85.

[10] Z. Yan zhao, M. Jian guo and S. Huan, "Research on Intelligent Vehicle Lane Keeping Based on SMC of Fuzzy Adaptive Method", Journal of Chong qing University of technology, vol. 24, no. 9, (2010), pp. 7-12.

[11] L. Wei, D. Jianmin and G. Jianwei, "Adaptive sliding mode control for uncertain systems with state time-delay and known external disturbances", Journal of Ji lin University, vol. 41, no. 1, (2011), pp. 249-253.

[12] Z. Ring-ming. R. Dian-bo, C. Sheng-min and Z. Ji-ye, "Direct adaptive control for lane keeping in intelligent vehicle systems", Journal of Harbin Institute of Technology, vol. 16, no. 6, (2009), pp. 810-814

[13] L. Yi nong, Y. Liu and Z. Ling, "Vehicle longitudinal and lateral coupling control based on sliding mode contro1", China Mechanical Engineering, vol. 18, no. 7, (2007), pp. 866-870.

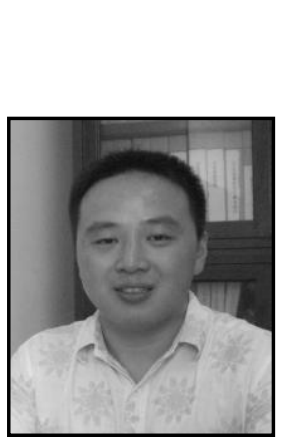

\section{Authors}

Junbo Zhao, (1979-) was born in Wangqing city of Jinin province of China and received his Bachelor degree in Automatic Control in 2002 from Chang'an University, Xi'an of China. After that, he received his major degree in software engineering from Talian University of technology, Dalian of China. He became a lecture in 2007 and has published more 10 papers and his current interest is traffic control and simulation.

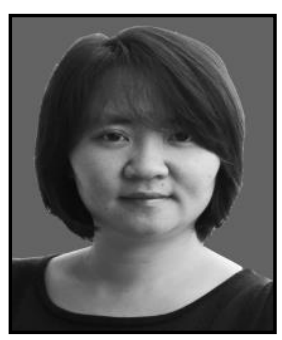

Jie Yang, (1980-) was born in Chongqing city of China and received her Bachelor degree in Automatic Control in 2002 from Chang'an University, Xi'an of China. After that, she received her major degree in traffic and transportation engineering from Tongji University, Shanghai of China. She became a lecture in 2007 and her famous book named drawing technology and project practice of architecture with Auto CAD has been pressed in China. Now she has published more 10 papers and her current interest is traffic control. 